\title{
Contemporary Chinese Youth's Reading Preference on Han Fu
}

\author{
Chen Qi \\ Foreign Language College, Chengdu University of Information Technology, China
}

Copyright $(2017$ by authors, all rights reserved. Authors agree that this article remains permanently open access under the terms of the Creative Commons Attribution License 4.0 International License

\begin{abstract}
The paper analyses the Han Fu's reading preference of contemporary young people in China. A questionnaire on the basis of Yang Xiong's four Fu, including two Big Fu and two Small Fu, and two Tang poems were designed and 200 non-Chinese major undergraduates in two universities were chosen out randomly to answer it. The investigation shows that most of the respondents do not like Small Fu but Big Fu. The author observes three special phenomena respectively in diction and rhythm, understanding, and length, and discovers they are closely connected with the prosperity of Chinese society and with the use of network. Moreover, compared with Han $\mathrm{Fu}$, most of those respondents like Tang poem because of the understanding and length which provides more evidences on the reasons of the special phenomena. The decline of the understanding ability of reading and the lack of reading patience caused by the use of network should be on the alert.
\end{abstract}

Keywords Reading Preference, Young People in China, Yang Xiong, Han Fu, Tang Poem

\section{Introduction}

Han $\mathrm{Fu}$ is the most popular style in Han Dynasty, accumulating precious experiences for the development of China's ancient literature. It is the starting point of ancient Chinese literary self-consciousness, greatly influenced by Chiu Yuan's (屈原) works and the free style in the Warring States Period. However, it had been mistaken as the synonym of "formalism" which carries a derogatory meaning in Chinese for its special historical and political background in a certain period of time. Fu didn't gain its due honor until the Third Plenary Session of the 11th Central Committee of the Chinese Communist Party. Chinese scholars gradually treat Fu objectively, sensibly and rationally. It can be divided into three kinds, Saoti Fu, Big Fu and Small Fu. Knechtges called Big Fu as "Fuh" and Small Fu as "Minor Poems". [1] Big Fu is usually long in length, huge in structure, and extremely gorgeous in diction. And Small Fu is short, plain and ironic, compared with Big Fu. Saoti Fu, as the beginning of $\mathrm{Han} \mathrm{Fu}$, is immature in form and style. Therefore, scholars draw their attention on the study of Big Fu and Small Fu, which are excessively fruitful in terms of quality and quantity. "But Yang Xiong's Fu is quite rarely explored, which remains a mystery for many oversea sinologists." [2] Knechtges calls Yang Xiong a "court poet in the late West Han Dynasty" and points out that though he is as important as Sima Xiangru (司 马相如) in the development of $\mathrm{Fu}$, Yang Xiong received much less attention. [3] There is a long way to go in the study of Yang Xiong's Fu. And this helps his works be the suitable Fu materials in the questionnaire designed by the author.

Since 1960s, the theory of literature acceptance represented by Wolfgang Iser and Hans Robert Jauss etc. has risen in former Federal Republic of Germany. It focuses on the reader's acceptance and emphasizes the relationship between text and the reader. The theory was introduced into China in 1980s. Chen Wenzhong points out that since then the development of literary acceptance has undergone three stages in China, which have been the introduction and assimilation stage, the study stage of acceptance history and the plural development stage.[4] Jauss believes the reader is the historical and dynamic element among the author, the works and the reader. [5] Iser says it is only in reading that the works can produce meaning. [6] Readers of different generations have different interpretations and preferences in the reading of the same works, because of their different growth environment, which is greatly molded by the society where they live. That's to say, human behaviors can reveal social environment. At the same time, the situation of a certain society could be anal sized according to its people's behavior, in which one of the important parts are language in various forms. Language is considered as "the bridge and mirror for people to learn or understand cultural value orientations". [7] David Block also says "linguistics, and any study associated with language, serves social analysis". [8] Literature works, as one of the decisive forms of language, plays a very important role in the study of society. Iser believes that literature works have their art and aesthetic, and art belongs to the authors' works while aesthetic belongs to 
the readers. [6] Since human beings are related to the society in which they live, some questions relating to human beings can be analyzed from the point of society's development and change. For Example, why do people from a certain society prefer to "this article" instead of "that article? What is the social background linked with such preference? These questions are worth researching. Some scholars points out the study of reading behavior will help them not only know people's ways of getting information, but also improve the pertinence and validation of reading promotion, since the change of reading behavior is one of the keys related to information explosion. [9] In fact, society's change with the development of economic, culture, environment, science and technology, could be found out from the way of getting information. The study of reading preference in a certain society (China) will reflect the society's situation to some degree.

\section{Research Materials and Design}

\subsection{Research Materials}

Four of Yang Xiong's Fu, two Tang poems and 200 undergraduates in two universities whose majors are not Chinese are the research materials.

The reasons why Yang Xiong's Fu were chosen are as follows. First, Yang Xiong, as one of the four most famous $\mathrm{Fu}$ writers in Han Dynasty, wrote down $\mathrm{Fu}$ in different styles, including Big Fu and Small Fu, which could make the research more accurate. Second, the study about Yang Xiong's Fu is not so widespread, while the study of the rest famous Fu writers' workers, such as Sima Xiangru's(司马 相如), Ban Gu's (班固) and Zhang Heng's (张衡) is excessively fruitful. So lots of work need to be done in the the research of Yang Xiong's Fu. Third, it would help the author observed the real responses of the 200 undergraduates in two universities, for they are unfamiliar with the content of Yang Xiong's Fu due to the academic attention's lack. In order to show the literary acceptance of $\mathrm{Han} \mathrm{Fu}$ in contemporary China clearly, two Tang poems are used in the study as the contrast materials. The samples are 200 non-Chinese major undergraduates in two universities. As everyone knows, students whose majors are Chinese, usually have higher abilities on Chinese reading and understanding than ordinary people. The aim of the paper is to try to study ordinary Chinese young people's reading preference on Han Fu. Therefore, they are not the suitable and objective samples for the research. And those non-Chinese majors undergraduates were chosen because they can be on behalf of the ordinary Chinese people's reading and understanding abilities since university education is now more and more popular.

\subsection{Research Design}

Four magnum opuses of Yang Xiong, including two Big $\mathrm{Fu}$ and two Small Fu were used in the study. Two Big Fu are “Gan Quan Fu” ( 《甘泉赋》) and “Chang Yang Fu” (《长杨赋》), while two Small Fu are "The Fu on Wine" (《酒赋》) and “Zhu Pin Fu” (《逐贫赋》). Two Tang peoms which is the most popular classical literature form are used in comparison. They are "Yang Liu Zhi” (《杨柳枝》) by Wen Tingjun (温庭筠), which describes the emperor's palace like many Han $\mathrm{Fu}$ do, and "Passing the Hua Qing Palace” (《过华清宫》) by Du Mu (杜牧), which describes the author's ironic psychology like "Zhu Pin Fu" of Yang Xiong. 20 students who did not take part in the later investigation were invited to read the four $\mathrm{Fu}$ at first and list the reasons why they like or not like the Big or Small Fu. Then, they will compare Han Fu with the two Tang poems and list the reasons why they like or not like $\mathrm{Han} \mathrm{Fu}$ or Tang poem. Their reasons were sorted out and classified into different items such as Like Big Fu and Not like Big Fu, Like Small $\mathrm{Fu}$ and Not Like Small Fu, Like Han $\mathrm{Fu}$ and Like Tang poem. The author used their answers as the options in a questionnaire according to the repetition rate and got rid of some useless reasons, such as "I just do not like it". 200 non-Chinese undergraduates in two universities were randomly selected out and answered the questionnaire after finishing their reading of the four $\mathrm{Fu}$ and two Tang poems mentioned above. In order to help them understand the meaning of Yang Xiong's four $\mathrm{Fu}$ and appreciate $\mathrm{Fu}$ 's diction and rhythm, translations in the vernacular besides the original and Chinese Pinyin to some unfamiliar words were offered at the same time. In the questionnaire, the students must answer the "like or not like" question, and then can answer different questions according to their different attitudes towards $\mathrm{Big} \mathrm{Fu}$ and $\mathrm{Small} \mathrm{Fu}$, and towards $\mathrm{Han} \mathrm{Fu}$ and Tang poems. Their answers were collected together and formed three tables to help the research.

\section{Results Analysis and Discussion}

\subsection{Questionnaire Results}

The author collected all the questionnaires from those 200 non-Chinese undergraduates mentioned above and formed Table 1, Table 2 and Table 3 as follows. One thing that is not shown in the Tables, is that all the respondents agree the four $\mathrm{Fu}$ are difficult to understand, and they just can choose the comparatively easy ones that they prefer to read. 
Table 1. Questionnaire Results on Reading Preference about "Like"

\begin{tabular}{|c|c|}
\hline Like Big Fu & 133 \\
\hline \multicolumn{2}{|l|}{ Reasons } \\
\hline Gorgeous in diction & 126 \\
\hline Rhythmic & 115 \\
\hline Big scenes description & 83 \\
\hline Easy to understand & 131 \\
\hline Pregnant with meaning & 26 \\
\hline Ingenious design & 57 \\
\hline Describe emperors' life & 2 \\
\hline Like Small Fu & 44 \\
\hline \multicolumn{2}{|l|}{ Reasons } \\
\hline Describe ordinary life & 22 \\
\hline Short in length & 38 \\
\hline Ingenious design & 37 \\
\hline Pregnant with meaning & 36 \\
\hline Easy to understand & 15 \\
\hline Gorgeous in diction & 5 \\
\hline Rhythmic & 7 \\
\hline Neutrality & 23 \\
\hline
\end{tabular}

Table 2. Questionnaire Results on Reading Preference about "Not Like"

\begin{tabular}{|c|c|}
\hline Not like Big Fu & $\mathbf{2 6}$ \\
\hline \multicolumn{2}{|c|}{ Reasons } \\
\hline Pile up words and phrases & 26 \\
\hline Not describe ordinary life & 17 \\
\hline Difficult to understand & 11 \\
\hline Too long in length & 23 \\
\hline Not like Small Fu & $\mathbf{7 6}$ \\
\hline Reasons & \\
\hline Difficult to understand & 75 \\
\hline Not strong sense of rhythm & 43 \\
\hline Neutrality & $\mathbf{9 8}$ \\
\hline
\end{tabular}

Table 3. Questionnaire Results on Reading Preference about "Han Fu" \& "Tang Poem"

\begin{tabular}{|c|c|}
\hline Like Han Fu & $\mathbf{1 3}$ \\
\hline \multicolumn{2}{|c|}{ Reasons } \\
\hline Gorgeous in diction & 13 \\
\hline Rhythmic & 11 \\
\hline Big scenes description & 3 \\
\hline Like Tang Poem & $\mathbf{1 8 5}$ \\
\hline Reasons & 185 \\
\hline Easy to understand & 179 \\
\hline Short in length & 21 \\
\hline Rhythmic & $\mathbf{2}$ \\
\hline Neutrality &
\end{tabular}

\subsection{Research Analysis}

\subsubsection{Reading Preference about "Like"}

Table 1 shows that $66.5 \%$ of the respondents like Big Fu, while only $22 \%$ like Small Fu. Most of those who like Big $\mathrm{Fu}$ express their preference on its strong sense of rhythm, gorgeous diction and easy understanding, compared with Small Fu. 131 choose its easy understanding, 126 choose its gorgeous in diction, and 115 are moved by its beautiful sense of rhythm. But not so many students focus on the Fu's meaning and design. 83 of these respondents confess they like Big Fu's description of big scenes, 57 say that they like its ingenious design, and 26 believe that it is pregnant with meaning. only 2 students say they like the description of emperors' life in the works. Easy understanding is the most popular reason. About Small $\mathrm{Fu}$, the reasons that the students choose most are to be pregnant with meaning, short in length and ingenious in design. There are 38 in the 44 respondents who like Small $\mathrm{Fu}$ choose the reason that it is short in length. It shows that essay's length is one of important factors when the respondents evaluate their reading preference. Students show little appreciation to its diction and rhythm, for only 12 of them choose the two items.

\subsubsection{Reading Preference about "Not Like"}

Although about half of the respondents remain neutral, Table 2 also shows that 26 respondents do not like Big Fu, and there are 76 do not like Small Fu. Almost all the people, who do not like Big Fu, agree that it is too long and piles up too many words and phrases to describe. 17 respondents do not like the content which is mainly about emperors' life, such as going hunting, going for an outing. Only 11 of them think it is difficult to understand, while 75 consider Small $\mathrm{Fu}$ as the difficult one in understanding. There are also 43 students think the sense of rhythm is not very strong in Small Fu. The two items above about Small Fu are the reasons why 76 respondents do not like it.

\subsubsection{Reading Preference about Han Fu and Tang poem}

Undoubtedly, contemporary Chinese young people much more like Tang poem. 185 of them prefer Tang poem, while only 13 prefer Han Fu. All the people who like Tang poem think it is easy to understand and 179 respondents like its short length. The main reasons to those who like Han Fu are its diction and rhythm.

\subsection{Research Discussion}

From the questionnaire, all the respondents think that the four $\mathrm{Fu}$ are difficult to understand. And it is obvious that people like Big Fu much more than Small Fu, and like Tang poem much more than Han Fu. Undoubtedly, some reasons cause the phenomenon. But three main reasons behind it could be found out. The first is the easy understanding the second is the gorgeous diction and strong rhythm. The third 
reason is the length. Therefore, the following discussion will focus on these three items - diction and rhythm, understanding, and length.

\subsubsection{About Diction and Rhythm}

Contemporary Chinese youth pay great attention on the aesthetic perception in literary works according to the questionnaire's results. There are 126 of the respondents said that they liked Big Fu for its gorgeous diction, and 115 for its rhythm. Meanwhile, 43 of all the 76 respondents who did not like Small Fu, expressed their discontent of its sense of rhythm. Han $\mathrm{Fu}$, especially $\mathrm{Big} \mathrm{Fu}$, is a type of literary works emphasizing the pursuit of beauty not only in voice but also in form. In voice, the beauty of $\mathrm{Fu}$ is usually composed of alliteration and assonance, mimetic words, etc.. And in form, the writers of $\mathrm{Fu}$ create various colorful artistic techniques of expression. For example, they decorate their works by the words full of color sensation and luminosity, which can make the readers have a deep impression. What's more, they are good at dualization bringing symmetrical beauty. Contemporary young people in China appreciate the strong sense of rhythm in Big $\mathrm{Fu}$, which enjoys them as music, and favor the form beauty such as symmetrical beauty. They consider diction and rhythm are the two important indexes in the evaluation of reading preference. That's why Big Fu is popular to the respondents. Thinking of the phenomenon that the respondents pay special attention to diction and rhythm, the author believes that social environment in China may be the reason. A person's observing and thinking ways is decided by the elements such as his or her values, ethics, and religion. [10] The social environment people live will have an influence on these elements, of course. Gong Kechang (龚克昌), a contemporary Chinese expert in Fu study, puts forward that though it's maybe not so splendid as Tang poetry, Song Ci, Yuan Qu and Ming and Qing's Novels, Han Fu demonstrates the reality of great Han dynasty's prosperity and describes Han's spirit and social atmosphere. [11] As the empire economy developed, the nation power was strong in Han Dynasty and provided a solid material foundation to the appearance and prosperity of $\mathrm{Fu}$. At the middle of Han Dynasty, Big $\mathrm{Fu}$ reached its historical climax with the development peak of nation power. At that time, people liked the colorful, rhythmic and ornamental Big $\mathrm{Fu}$ which revealed strong nation power, consolidated regime and social abundance, and also shows people's high self-confidence for the advantageous social conditions. If we focus on the social environment of Han Fu's climax and today's China, some similar points appear. For example, today's China has consolidated regime and social abundance, too. Nation power is improved greatly, compared with 100 years ago. And with the development of economy and the improvement of living standard, contemporary Chinese become more and more self-confident. It may be reasonable that contemporary young people in China appreciate the aesthetic perception in
Big Fu's diction and rhythm.

\subsubsection{About Understanding}

131 of all the 133 respondents agree that $\mathrm{Big} \mathrm{Fu}$ is easier to understand than Small $\mathrm{Fu}$ and this is one of the most important reasons why they like it. In fact, almost all the people like Big Fu choose this reason. Meanwhile, 75 of all the 76 respondents who dislike Small Fu think it is too difficult to understand. Furthermore, all the respondents agree the four $\mathrm{Fu}$ are difficult to understand, compared with Tang poem and this is one of the two main reasons why they much more like Tang poem. The ease of understanding is one of the vital elements in the respondents' reading preference. Although no one would like the written materials that cannot be understood, it is inevitable to show the decline in the understanding ability of reading belonging to contemporary young people in China. The author believes that the decline is closely related with the network environment the Chinese young people are facing. Reading behaviors are changing in nowadays new environment. Digitization and hypertext fragmentation in digital reading are caused the decline of the understanding ability of reading. Ramirez discovers that $68 \%$ of the respondents believe they can understand and remember more information by reading printed books than e-books. [12] And Zhao Shanshan thinks it is because people can make some notes more conveniently, if they read the printed books. [13] Jeong gets the similar conclusion that reading printing books are more helpful to the understanding ability than reading e-books in the study of 6-year-old children. [14] Although Jeong's respondents are children, the inclination for adults probably is the same. All of these prove the reading of e-materials is bad for people's understanding ability of reading, compared with the reading of printing materials. But the fact is that young people spend more and more time in surfing the webpage. The investigation made by Ismail and Negah proved that young people, especially students are those who use network more frequently than others in a society. [15] Moreover, webpage's' readers have some characters. Some Scholars find out one of these characters is that such readers incline to scan the web pages and read only about $20 \%$ of the text for each webpage on the average. [16] Such scanning means the inevitable lack of content to some degree, which has a negative influence on the deep understanding of the content. If this inclination exists for a comparatively long time, young people would meet some problems in the understanding ability to the written materials. Birkerts considers that the younger generation growing up in the digital environment is lack of the reading ability in depth. [17] Besides, the features of openness and fastness of network media decide that network culture is a kind of "fast food culture". This kind of culture lacks cultural and ideological content, and encourages young people to seek quick success and instant benefits in reading. If they read a little difficult reading material, young people under the "fast food" culture, will 
naturally avoid spending their time to study and understand the meaning, for the reading cannot give them quick success. That's why the respondents' understanding ability of reading have declined and why the respondents in the paper show their strong preference in the ease of understanding.

\subsubsection{About Length}

One of the results in questionnaire is about the length. In the reading preference showing "Like" of Fu, 38 in all the 44 respondents who like Small Fu express their appreciation of its shortness in length, compared with Big Fu. In the reading preference showing "Not Like" of Fu, $88 \%$ who do not like Big Fu consider that it is too long. At the same time, 179 in all the 185 respondents who like Tang poem appreciate its short length, compared with Han Fu. It is an interesting phenomenon that an essay's length becomes a comparatively more important element than ever in reading, when people evaluate their own reading preference. The author believes the phenomenon probably is caused by the impact of network culture, too. As the author mentions above, most of the contemporary young people in China have already closely connect their daily reading with network by using mobile phones or computers, with the development of science and technology. Annual Report in Digital Publishing Industry in China 2014-2015 shows the revenue of digital publishing is more than 330 billion RMB in 2014 and increased 33.36\%. [18] The digital reading has already become the most popular reading way in China. Every essay's reading time are reduced due to the huge amount of written materials and the limit of time. [19] Some Scholars also discover that readers spend only about 4 minutes in reading e-books and only 8 minutes in reading electronic journals on the average. [20] It shows that readers try to use scanning more often in order to obtain a large number of useful information as quickly as possible. And it is well-known that the longer the essay is, the more time they use. From the point of learning information quickly and effectively in a short time, it is naturally to avoid choosing long written materials to read in order to save time and energy. Therefore, young people in China prefer the shorter $\mathrm{Fu}$ ( $\mathrm{Small} \mathrm{Fu}$ ) to the much longer $\mathrm{Fu}$ (Big Fu), and the shorter Tang poem to the longer Han Fu.

One more thing need to be pointed out that though the length is one of the key elements, the ease of understanding is more important in evaluating Chinese young people's reading preference of $\mathrm{Fu}$, according to the investigation.

\section{Conclusions}

Reading, as one of the basic paths of gaining knowledge and inheriting culture, plays a very important role not only in the personal growth and development but in the development of society and culture. Meanwhile, the study of reading preference can help people know society's change from another point of view, which make the study meaningful. The study of $\mathrm{Han} \mathrm{Fu}$, a highly ornamental and much-touted literary form, has caught more and more scholars' eyes in recent years. But Yang Xiong, as one of the most outstanding and accomplished Fu writers in Han Dynasty, still lacks academic attention. So the author chooses his works as the reading materials in the investigation. To obtain more evidences, the author also chooses two Tang poems as the contrast sample. From the 200 non-Chinese undergraduates' responses, three items about diction and rhythm, understanding, and length are paid special attention in Fu's reading preference. The reading preference about diction and rhythm shows the self-confidence with the present prosperity of Chinese society, similar with Han Dynasty. The reading preference about understanding and length proves the profound influence of network on the younger generation in China today. But what needs to be vigilant is the decline of the understanding ability of reading and the lack of reading patience among young people in China under the Internet environment.

\section{Acknowledgements}

This essay is an achievement of the project "Analysis of the Heterogeneities in Sino-US Yang Xiong Researches from Perspective of Comparative Literature" (SC16WY005) supported by Sichuan Federation of Social Sciences Circle.

\section{REFERENCES}

[1] David R. Knechtges.Yang Shyong, The Fuh, And Hann Rhetoric. University of Washington, Washington, 1968.

[2] Lu Jie. Divergences in American and Chinese Scholars' Researches on Yang Xiong's Fu. Linguistics and Literature Studies, 2017, 5(2), 84-90.

[3] David R. Knechtges. Studies of Han Dynasty Court Literature and Culture. Trans. Su Ruilong. Shanghai Translation Publishing House, Shanghai, 2013.

[4] Chen Wenzhong. Reviews and Reflections on the Study of the History of Literary Reception in 20 Years. Journal of Anhui Normal University (Hum. \& Soc .Sci .), 2003(5): 534-543.

[5] Hans Robert Jauss. Zhou Ning \& Jin Yuanpu (trans.) Reception Aesthetics and Reception Theory. Liaoning People's Publishing House, Shenyang, 1987.

[6] Iser-Wolfgang. The Act of Reading, A Theory of Aesthetic Response. The Johns Hopkins University Press, Baltimore and London, 1987.

[7] Lucy Xing Lu \& Guoming CHEN, Language Change and Value Orientations in Chinese Culture, in: Steve J. Kulich, Liping Weng \& Michael H. Prosser (Eds.), Value dimensions and their contextual dynamics across cultures, Shanghai Foreign Language Education Press, Shanghai, 2014, 285-304. 
[8] David Block, The Social Turn in Second Language Acquisition, Shanghai Foreign Language Education Press, Shanghai, 2016.

[9] Shabani, A., F. Naderikhara and M. Abedi. Reading Behavior in Digital Environments among Higher Education Students. Library Review, 2011, 60(8), 645-657.

[10] Chen Qi. The Influence of Chinese Social Action Orientation on Chinese-English Trademark Translation. Journal of Changchun Institute of Technology (Social Sciences Edition), 2016(3), 60-62.

[11] Gong Kechang. A Study on Chinese Fuh. Shandong: Shandong University Press, Jinan, 2010.

[12] Ramirez, E. The Impact of the Internet on the Reading Practices of a University Community: the Case of UNAM. New Review of Libraries and Lifelong Learning, 2003, 4(1), 137-157.

[13] Zhao Shanshan. A Review of Research on Digital Reading Behavior in Foreign Countries. Library Forum, 2015(10), 118-124.

[14] Jeong, H., A Comparison of the Influence of Electronic Books and Paper Books on Reading Comprehension, Eye
Fatigue, and Perception. The Electronic Library, 2012, 30(3), 390-408.

[15] Ismail. R. and Z. Negah. The Pattern of E-book Use amongst Undergraduate in Malaysia: A Case of to Know is to Use. Malaysian Journal of Library \& Information Science, 2005, $10(2), 1-23$.

[16] Weinreich, H., H. Obendorf, E. Herder, et al. Not Quite the Average: An Empirical Study of Web Use. ACM Transactions on the Web, 2008, 2(1), 1-31.

[17] Birkers, S. The Gutenberg Elegies: The Fate of Reading in an Electronic Age. Faber \& Faber, Boston, 2006.

[18] Annual Report in Digital Publishing Industry in China 2014-2015 shows the revenue of digital publishing is more than 330 billion RMB. Online available from http://www.gapp.gov.cn/news/1656/256063.shtml

[19] Tenpir. C., D. King, S. Edwards and L.Wu. Electronic Journals and Changes in Scholarly Article Seeking and Reading Patterns. Aslib Proceeding, 2009, 61(1), 5-32.

[20] Rowlands, I, D.Nicholas, P. Williams, et al. The Google Generation: The Information Behaviour of the Researcher of the Future. Aslib Proceedings 2008, 60(4), 290-310. 